\title{
Mapeamento de informações organizacionais: um estudo na Embrapa
}

\author{
Organizational information mapping: a study at Embrapa
}

Eduardo Amadeu Dutra MORESI 1

Rosana Guedes Cordeiro RAMOS 2

Hércules Antônio do PRADO ${ }^{3}$

\section{RE S U M O}

As informações armazenadas nos sistemas corporativos são, geralmente, desconhecidas pela maioria dos membros de uma organização. Muitas vezes a informação necessária está disponível, mas as pessoas não sabem como acessá-la e nem da sua existência. Diante desta incógnita, tratou-se a seguinte questão: como identificar as informações de uma organização a partir dos seus sistemas de informação? Para resolvê-la, realizou-se uma pesquisa qualitativa, metodológica e documental, visando definir um mapa das informações armazenadas em sistemas de informação corporativos da Embrapa. Para a construção do mapa, definiu-se um processo para sistematizar o mapeamento das informações. O processo baseou-se em conceitos da arquitetura da informação, de representação por meio de metadados, de classificação das informações e de correlação com os processos de negócio da empresa. A aplicação do processo proposto foi feita em três sistemas corporativos da Embrapa. Foram analisados sessenta e três relatórios para a construção do mapa de informação. Foi possível responder às seguintes questões: quais informações estão duplicadas na organização e onde elas estão; quais relatórios têm excesso de informações; quem pode ter acesso a determinada informação; quais são os processos de negócios apoiados por determinado sistema de informação. Concluiu-se que apenas a consulta disponível em sistemas informatizados não é suficiente para ter acesso à informação. A construção de um mapa de informações permite facilitar a localização e o acesso às informações armazenadas nos diversos sistemas corporativos.

Palavras-chave: Arquitetura da informação. Gestão da informação. Mapeamento de informações. Metadados.

\section{A B S T R A C T}

Information stored in computer systems are, generally, unknown by most of the members of an organization. Frequently, information needed is available, but people do not know how to access it and about its existence. Having that issue in mind, the following question was posed: how to identify an organization information in its

\footnotetext{
1 Professor Doutor, Universidade Católica de Brasília, Curso de Ciência da Computação. QS 7, Lote 1, Águas Claras, 71966-700, Brasília, DF, Brasil. Correspondência para/Correspondence to: E-mail: <moresi@ucb.br>.

2 Analista de Sistemas, Empresa Brasileira de Pesquisa Agropecuária, Departamento de Tecnologia da Informação. Parque Estação Biológica. Brasília, DF, Brasil.

3 Analista, Empresa Brasileira de Pesquisa Agropecuária, Secretaria de Gestão Estratégica. Brasília, DF, Brasil. Recebido em 19/4/2009 e aceito para publicação em 15/7/2010.
} 
information systems? To answer it, a qualitative, methodological and documental research was carried out to define a map of the information stored in the corporate information systems of Embrapa (Brazilian Agricultural Research Corporation). To build the map, a process to systemize the mapping of information was created. The process was based on information architecture concepts, metadata representation, classification of information and relationship with the enterprise business process. The proposed process was applied in three corporate systems of Embrapa. To create the information mapping, sixty three reports were analyzed. Using the map some answers could be answered to improve the decisions, such as: "what information are duplicated in the organization and where are they?"; "which reports have much information?"; "who can access some information?"; "which are the business processes supported by an information system?" It can be concluded that queries available on information systems are not enough to have access to information. An information map facilitates localization and access to the information stored on different corporate systems.

Keywords: Information architecture. Information management. Information mapping. Metadata.

\section{INTRODUÇÃ O}

A maioria das organizações utiliza sistemas de informação para automatizar seus processos de trabalho, para armazenar e recuperar dados e para compartilhar informações. Na chamada era da informação, gerenciar adequadamente as informações é fator essencial para o sucesso da organização (Wetherbe, 1986; Davenport, 1998; Moody, 1999; Rosini, 2003).

A informação passou a ser tratada como um bem da organização: a informação certa, no formato adequado e na hora ideal pode proporcionar boas oportunidades de negócios. A necessidade de gerenciar estas informações e disponibilizá-las adequadamente levou a grandes investimentos em tecnologia, acreditando-se que computadores e ferramentas poderiam ser a solução para esse problema (Davenport, 1998).

Sistemas de informação estão sendo cada vez mais utilizados para se obter vantagem competitiva. Porém, somente a tecnologia não basta para o sucesso de uma organização na era da informação, isło é, todos os computadores do mundo de nada servirão se seus usuários não estiverem interessados na informação que estes computadores podem mostrar (Davenport, 1998).

Através da ecologia da informação é possível dar mais ênfase ao ambiente informacional da organização, pois essa abordagem baseia-se em como as pessoas criam, distribuem, compreendem e usam a informação. A tecnologia é colocada como um meio para disseminar informações e dados, e não como fator essencial num processo de gestão da informação. Essa visão ecológica é subdividida em três ambientes: ambiente informacional, ambiente organizacional e ambiente externo. A arquitetura da informação, principal foco deste trabalho, faz parte do ambiente informacional da organização.

A arquitetura da informação, que é um dos componentes do ambiente informacional da organização, pode ser definida como um guia para estruturar e localizar a informação dentro de uma organização. Uma das ferramentas mais simples para a aplicação da arquitetura da informação é o mapa de informações (Davenport et al., 2004), que indica aos membros de uma empresa onde se encontram tipos específicos de informação. Porém, esses mapas são raros nas organizações, o que evidencia o quanto a gestão da informação é pouco praticada nas empresas.

Essa pesquisa tem como objetivo o mapeamento das informações contidas nos sistemas de informações de uma organização. O mapeamento de informações está inserido no contexto da gestão da informação, mais especificamente no eixo da arquitetura da informação e é focalizado na utilização da informação no presente, ou seja, aquela que se tem hoje e que é utilizada pela organização (Davenport, 1998).

Acredita-se que, a partir deste mapeamento, as informações poderão ser mais bem utilizadas na organização. Os sistemas de informação, que as disponibilizam, poderão servir como apoio aos processos de tomada de decisão da empresa. Dessa forma, será uma solução para compartilhar e disseminar mais rapidamente as informações existentes na organização.

Nesse sentido, este artigo apresenta uma aplicação da arquitetura da informação para mapear as informações dos sistemas de informação de uma 
organização, a fim de possibilitar uma melhor localização dessas informações. Um estudo de caso foi desenvolvido na Empresa Brasileira de Pesquisa Agropecuária (Embrapa), que resultou na definição de um processo para mapeamento de informações existentes em sistemas de informação.

\section{A RQUITETURA DA INFORMAÇÃO}

A arquitetura da informação é um guia para estruturar e localizar a informação dentro de uma organização (Davenport, 1998). É um modelo que mostra como as categorias de informação estão relacionadas aos processos de negócio da organização, e como essas categorias necessitam estar conectadas para facilitar o suporte aos tomadores de decisão (Wetherbe, 1986). É uma planta arquitetural para modelar os requisitos de informação global de uma organização. Proporciona uma forma para mapear as necessidades de informação de uma empresa.

Um dos maiores objetivos da arquitetura da informação é fornecer uma estrutura lógica para ajudar a encontrar a informação de que se necessita (Rosenfeld; Morville, 2002). A sua definição compreende:

- a combinação de organização, nomeação e esquemas de navegação juntamente com um sistema de informação;

- o projeto estrutural de um espaço informacional para facilitar a conclusão de tarefas e o acesso intuitivo ao conteúdo; e

- a arte e a ciência de estruturar e classificar web sites e intranet para auxiliar as pessoas a encontrar e gerenciar a informação.

A arquitetura da informação focaliza a informação em todas as suas formas e tamanhos, como por exemplo: web sites, documentos, softwares aplicativos e imagens. Engloba, também, os metadados - termos utilizados para descrever e representar o conteúdo dos objetos como documentos, pessoas, processos e organizações. $\bigcirc$ foco principal da arquitetura da informação é tornar acessível o que já existe na organização.
Para esta pesquisa adotou-se o seguinte conceito para arquitetura da informação: conjunto de princípios, diretrizes, padrões e procedimentos que visam estruturar, organizar, qualificar, localizar e gerenciar a informação existente na organização.

Estruturar significa determinar os níveis de granularidade para as informações, ou seja, definir o espaço informacional ${ }^{4}$ a ser tratado. Organizar envolve o agrupamento de componentes, identificados no espaço informacional, em categorias principais e distintas. $E$ qualificar significa designar como chamar cada categoria de informação organizada.

O avanço tecnológico e o aumento significativo de sistemas de informação requerem que a organização tenha total domínio e controle sobre seu recurso informacional. A informação deve estar disponível onde e quando é solicitada. As empresas que não reconhecerem isso estarão em desvantagem competitiva, tendendo ao insucesso (Wetherbe, 1986).

Qual é a importância da arquitetura da informação para uma organização? Por que investir em uma arquitetura da informação? Qual o retorno desse investimento? Ao se questionar esses aspectos (Rosenfeld; Morville, 2002), deve-se considerar o seguinte:

- o custo de encontrar a informação: "Quanto custará se cada empregado da sua organização passar mais de cinco minutos por dia para encontrar uma informação de que precisa?" e

- o custo de não encontrar a informação: "Quantas más decisões são tomadas por dia na sua organização por que os empregados não encontram as informações de que eles necessitam?".

\section{MÉTODOS}

Essa pesquisa classifica-se quanto aos fins como sendo qualitativa, metodológica e aplicada. De acordo com Moresi (2003) uma pesquisa qualitativa considera que há uma relação dinâmica entre o mundo real e o sujeito, isto é, um vínculo indissociável entre o mundo objetivo e a subjetividade do sujeito que não pode ser traduzido em números. $\bigcirc$ processo e seu significado

\footnotetext{
4 Para espaço informacional entendem-se os sistemas de informação automatizados, os documentos escritos, jornais, artigos, relatórios, formulários, parágrafos ou sentença, diretrizes e normas da empresa, informações em vídeo ou áudio, ou necessidades de informações futuras (planejamento estratégico de informações).
} 
são os focos principais de abordagem. É uma pesquisa metodológica, pois se trata de um estudo relativo à elaboração de um instrumento de captação da realidade. Está associada a caminhos, formas, maneiras, procedimentos para atingir determinado fim. $O$ instrumento de captação é o mapa de informação elaborado. É uma pesquisa aplicada, pois existe a necessidade de resolver um problema concreto, com finalidade prática, no caso, localizar as informações existentes numa organização a partir de seus sistemas de informação.

Quanto aos meios, esta pesquisa é classificada como documental e bibliográfica. De acordo com Moresi (2003) a investigação documental é aquela realizada em documentos conservados no interior de órgãos públicos e privados, de qualquer natureza, ou com pessoas: registros, anais, regulamentos, circulares, ofícios, memorandos, balancetes, comunicações informais e outros. Nesta pesquisa estaremos utilizando os sistemas de informações da Embrapa e suas respectivas bases de dados.

A pesquisa bibliográfica, segundo Lakatos e Marconi (2003), é um apanhado geral sobre os principais trabalhos já realizados, revestidos de importância, por serem capazes de fornecer dados atuais e relevantes relacionados com o tema de pesquisa. Fornece instrumental analítico para qualquer outro tipo de pesquisa, mas também pode esgotar-se em si mesma. O material publicado pode ser fonte primária ou secundária.

A fase de levantamento de dados da pesquisa foi executada utilizando dois procedimentos: pesquisa bibliográfica e pesquisa documental. Para pesquisa bibliográfica foi feito um apanhado geral dos trabalhos realizados e relevantes com o tema desta pesquisa. $\mathrm{A}$ partir deste apanhado verificou-se que não existiam procedimentos relatados a respeito de como fazer um mapeamento de informações. Ainda na pesquisa bibliográfica identificaram-se na literatura quais seriam os pilares teóricos que pudessem subsidiar a realização e finalização da pesquisa.

Assim, definiram-se os limites da pesquisa, em relação ao assunto que foi arquitetura da informação e, em relação à extensão, que foi o mapeamento das informações dos sistemas de informação. Após a elaboração do Processo de Mapeamento de infor- mações, esse foi aplicado na Embrapa, seguindo todas as atividades e tarefas descritas, mapeando três sistemas de informação corporativos ${ }^{5}$.

\section{O MAPA DE INFORMAÇÕES}

Com o crescente avanço tecnológico, muitas organizações têm automatizado seus processos de negócio e criado sistemas de informação para armazenar e compartilhar suas informações, a fim de se tornarem cada vez mais eficientes e eficazes na execução de suas atividades (Laudon; Laudon, 2004). Um grande esforço por parte da área de Tecnologia da Informação das organizações é dispendido para atender às necessidades de informações dos usuários, vários sistemas são colocados em produção, uma enorme quantidade de dados está armazenada nos bancos de dados, web sites são construídos para disponibilizar informações, e mesmo assim, existe uma grande dificuldade em encontrar a informação de que se necessita.

Muitas vezes os gerentes recorrem a outras pessoas para saberem como encontrar uma determinada informação e não aos sistemas de informação existentes. Por que isto? Em geral não é de seu conhecimento quais as informações que são tratadas por um determinado sistema. Como não saber, se o sistema está disponível para vários usuários da empresa. Por não acharem o que necessitam nos sistemas de informação organizacionais, os gestores de negócio utilizam frequentemente uma rede informal de contatos, perguntando a pessoas do mesmo grupo sobre o que precisam, obtendo assim uma resposta em tempo hábil (Prusak; McGee, 1994). Em média os gerentes gastam $50 \%$ do seu tempo procurando informações de que eles precisam para executar suas atividades (Wetherbe, 1991).

A maioria dos sistemas de informação desenvolvidos nas organizações tem como objetivo atender às necessidades do nível operacional da empresa, oferecendo relatórios detalhados ou listas com dados relacionados (Laudon; Laudon, 2004). As informações desses sistemas ou estão subentendidas nesses relatórios ou sob domínio dos especialistas que desenvolveram o sistema.

\footnotetext{
5 São sistemas de uso na organização como um todo, que se integram em bases de dados corporativas e podem prover suporte para as atividades em todos os níveis gerenciais: estratégico, tático e operacional (Embrapa, 2002).
} 
Por não saberem quais são as informações tratadas nos sistemas organizacionais, pressupõe-se que os usuários não as utilizam adequadamente, não fazem com que sejam instrumentos de apoio para os processos de tomada de decisão da empresa, ou seja, os sistemas são subutilizados e, na maioria das vezes, deixados de lado. Isso ressalta a importância de um mapa para que se possa identificar o caminho a partir da informação (Wurman, 1991), que diga ao usuário onde ele está em relação à informação.

Os mapas são os meios metafóricos através dos quais se pode entender a informação que vem de fontes exteriores. Para criar um mapa de informações utilizando os conceitos da arquitetura da informação, tornou-se necessária a definição de um processo para orientar e padronizar a execução das atividades para elaboração do mapa.

processo de mapeamento das informações dos sistemas de informação foi definido de acordo com a metodologia de Análise e Melhoria de Processos da Embrapa (Embrapa, 2002) e foi baseado no processo para criação de uma arquitetura da informação proposto por Rosenfeld e Morville (2002).

\section{Descrição do processo de mapeamento das informações dos sistemas de infor- mação}

O Processo de Mapeamento de informações dos sistemas de informação foi subdividido em três subprocessos que são: planejamento, elaboração e manutenção do mapa de informações (Figura 1).

Planejamento do mapa de informações: esse subprocesso tem como objetivo fazer um planejamento do que se deseja mapear na organização, ou seja, decidir quais sistemas de informação farão parte do mapa de informações a ser construído, assim com também definir a estrutura do mapa de informações da organização. É composto pelas seguintes atividades: definir o espaço informacional a ser mapeado; elaborar uma descrição básica dos sistemas de informação do espaço informacional.

Elaboração do mapa de informações: esse subprocesso tem como objetivo alimentar a estrutura do mapa de informações definida no subprocesso de Planejamento. Suas atividades devem ser executadas para cada sistema a ser mapeado. É composto pelas seguintes atividades: identificar o(s) processo(s) de negócio do sistema de informação; relacionar as áreas da organização que utilizam o sistema de informação; familiarizar-se com o sistema de informação a ser mapeado; definir o conteúdo a ser investigado para o mapeamento; analisar o conteúdo informacional para identificação das informações disponíveis; categorizar as informações identificadas; alimentar a estrutura física do mapa de informações; validar a categorização de informações realizada; validar o mapa de informação construído; disponibilizar o mapa de informações para ser utilizado na organização.

Manutenção do mapa de informações: esse subprocesso tem como objetivo manter o mapa de informações atualizado para garantir que as mudanças efetuadas nos sistemas de informação estejam refletidas no mapa. Toda modificação de necessidades de informação dos sistemas mapeados deve ser encaminhada para o arquiteto da informação poder atualizar o mapa de informações. Nesse caso o processo continuará a partir da atividade de Análise do conteúdo informacional, do subprocesso de Elaboração do mapa. É composto pelas seguintes atividades: receber e analisar as atualizações de informações; atualizar a estrutura do mapa de informações.

processo elaborado contém para cada atividade: definição, objetivo, o que fazer, responsável pela atividade, produto de entrada e produto de saída. A aplicação do processo tem como produto final o mapa de informações dos sistemas de informação da organização.

\section{Estrutura do mapa de informações}

Nesta pesquisa, a estrutura definida para o mapa de informações está baseada no modelo proposto por Zachman (1992), que descreve os seis elementos básicos para uma arquitetura empresarial: o que (what), como (how), onde (where), quem (who), quando (when) e por que (why). Esses elementos são respectivamente: dados, processos, localização, pessoas, tempo e motivação. $\bigcirc$ modelo é uma estrutura lógica e compreensível para descrever a representação de qualquer objeto complexo e é neutro em consideração aos processos e ferramentas utilizadas para produzir as descrições. Desse modelo foram selecionados os seguintes elementos para compor o mapa de informações, que são: o que (what) - que corresponde às informações; como (how) - quais os processos de 


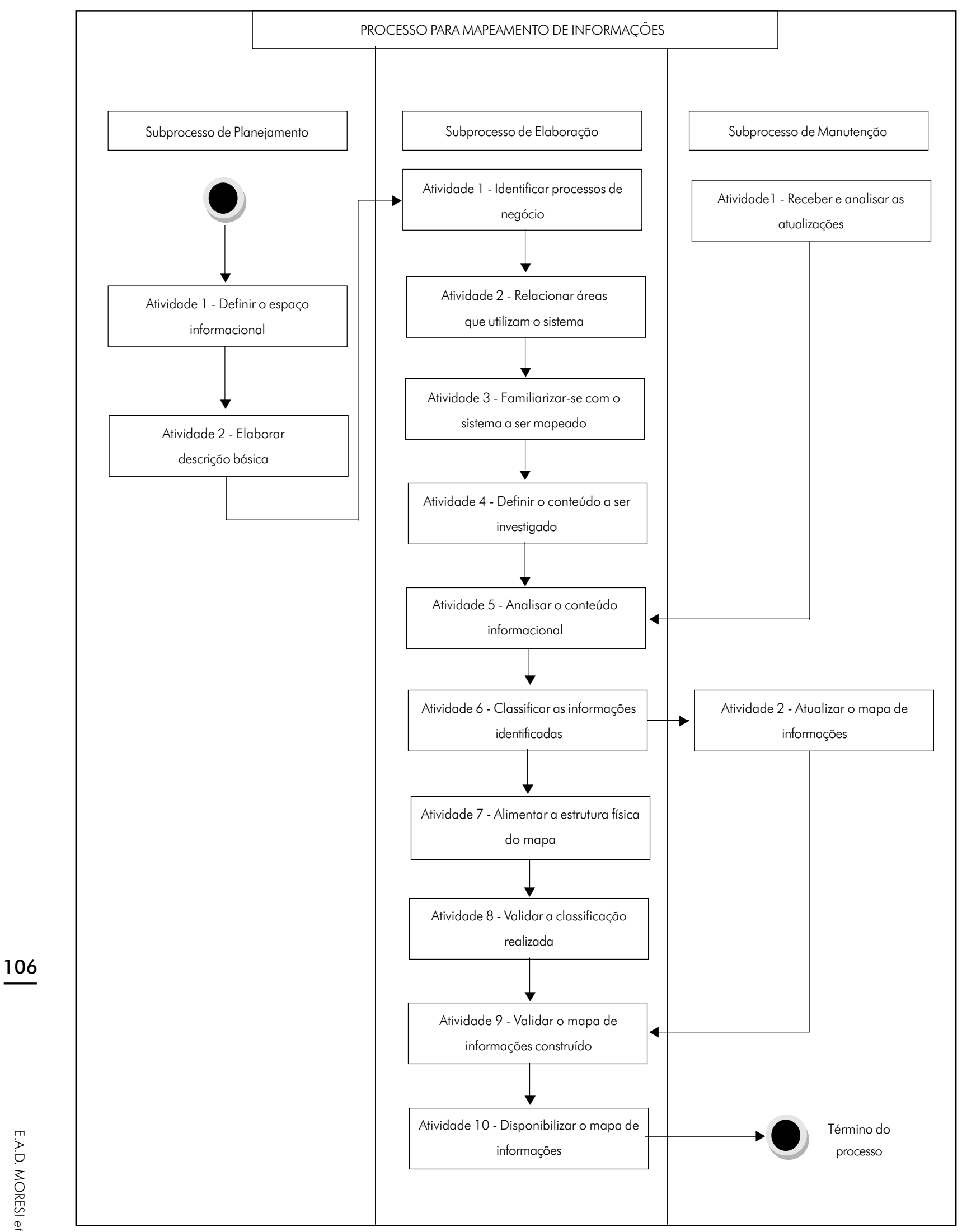

Figura 1. Diagrama de atividades do processo de mapeamento de informações.

TransInformação, Campinas, 22(2):101-110, maio/ago., 2010 
negócio a informação está associada; onde (where) local onde as informações podem ser encontradas; e quem (who) - que corresponde a qual área da organização é responsável pelo sistema de informação.

Está baseado também em Wetherbe (1986), que faz a correlação entre as categorias de informação com os processos de negócio da organização. De acordo com Rosenfeld e Morville (2002) deve-se procurar por um esquema de classificação público ou um tesaurus para a construção de uma arquitetura da informação. Com base nessa afirmativa optou-se por utilizar uma classificação já existente na organização, no que diz respeito à classe, à subclasse e ao subgrupo da informação. Portanto, a classificação adotada no mapa de informações proposto baseia-se no Código de Classificação de Documentos de Arquivo da Embrapa (Embrapa, 2003). A utilidade de um esquema de classificação é possibilitar uma concentração focada nos aspectos selecionados de um objeto sem perder o contexto (Zachman, 1992).

Através desse estudo foram identificados quais seriam os metadados (Alcântara et al., 2004) mais relevantes para o mapa de informações, a fim de tornar as informações do espaço informacional acessíveis. Sendo assim os metadados definidos foram:

- Classe da informação: as classes correspondem ao agrupamento das grandes funções desempenhadas pela empresa, ou seja suas atividades fim e meio;

- Subclasse da informação: agrupamento das informações subordinadas a uma classe de informação;

- Subgrupo da informação: subgrupo de uma subclasse da informação criado para detalhar a categoria da informação;

- Informação existente: informação contida no sistema de informação;

- Processo de negócio: refere-se ao processo de negócio ao qual a informação está associada;

- Unidade responsável: unidade organizacional responsável pelo sistema de informação;

- Localização da informação: em qual sistema a informação pode ser encontrada e em que local do sistema, podendo ser: numa tela de consulta, um relatório, manual do sistema, manual do usuário ou página da intranet. A mesma informação pode estar em mais de um local;
- Condições de acesso: pré-requisito para acessar a informação, qual o perfil de usuário necessário para acessar a informação.

\section{RESULTADOS}

O processo de mapeamento foi aplicado a três sistemas corporativos da Embrapa, cuja descrição resumida é apresentada no Anexo 1. Os sistemas incluídos no espaço informacional mapeado foram os seguintes: Sistema de Planejamento, Acompanhamento e Avaliação de Resultados do Trabalho Individual (SAAD); Sistema de Planejamento de Atividades da Embrapa (SISPAT); Sistema de Eventos da Embrapa (SIEVE).

Nos três sistemas mapeados foram identificadas 92 informações resultantes dos relatórios e consultas disponíveis em cada um dos sistemas. Um exemplo da estrutura do mapa de informações construído é apresentado no Anexo 2, onde se têm dados reais do estudo realizado na Embrapa. A partir desse mapeamento identificou-se que o Código de Classificação de Documentos de Arquivo (CCDA), utilizado pela Embrapa é adequado para se utilizar na categorização das informações constantes nos sistemas de informação.

Das 92 informações mapeadas foram encontrados: sete casos da mesma informação em relatórios e consultas diferentes no mesmo sistema; 13 casos do mesmo relatório atendendo a diferentes necessidades de informação; 12 casos de informações em subgrupos de informação diferentes; três casos da mesma informação em sistemas diferentes; e 14 casos de informações que não foram classificadas em um subgrupo específico. $\bigcirc$ Anexo 3 apresenta uma síntese do resultado obtido após a elaboração do mapa de informações.

\section{CONCLUSÃO}

A partir de uma revisão de literatura realizada verificou-se a ausência de trabalhos que relatassem a respeito da aplicação da arquitetura da informação nas organizações. Diante desta lacuna, foi desenvolvida uma pesquisa para definir um processo para a construção de mapas de informações a partir de sistemas 
de informações da organização. Esta construção baseou-se em investigação documental para definição dos respectivos metadados. $O$ processo proposto tem como produto final um mapa de informações a ser utilizado como um mecanismo de localização de informações em sistemas corporativos.

Para o sucesso de implantação do processo de mapeamento, recomenda-se: divulgar a importância da arquitetura da informação e do mapa de informações para a organização, além de seus benefícios; atribuir a função de mapeamento a um profissional da área de informação, que deverá estar dedicado exclusivamente à execução das atividades do processo de mapeamento; definir uma diretriz no processo de desenvolvimento de sistemas da organização para quando houver modificação das necessidades de informações de um sistema, que esta seja comunicada ao arquiteto da informação para atualização do mapa de informações

\section{REFERÊNCIAS}

ALCANTARA, A.; MORESI E.A.D.; PRADO, H.A. Metadados: conceito e uso expandidos. In: CONGRESSO ANUAL DA TECNOLOGIA DA INFORMAÇÃO, 2004, São Paulo. Anais... São Paulo: Fundação Getúlio Vargas, 2004.

DAVENPORT, T.H. Ecologia da informação: por que só a tecnologia não basta para o sucesso na era da informação. São Paulo: Futura, 1998.

DAVENPORT, T.H.; MARCHAND, D.A.; DICKSON, T. Dominando a gestão da informação. Porto Alegre: Bookman, 2004.

EMPRESA BRASILEIRA DE PESQUISA AGROPECUÁRIA (Brasilia, DF). Guia para categorização de produtos de software da Embrapa. Brasília: Embrapa, 2002.

EMPRESA BRASILEIRA DE PESQUISA AGROPECUÁRIA (Brasilia, DF). Código de classificação de documentos de arquivo. Brasília: Embrapa, 2003.

HAGEDORN, K. Extracting value from automated classification tool: the role of manual involvement and controlled vocabularies. Argus Associates, March, 2001. Available from: <http:// www.argus-acia.com>. Cited: 5 Oct. 2004.

LAUDON, K.; LAUDON, J. Sistemas de informação gerenciais: administrando a empresa digital. São Paulo: Prentice Hall, 2004.

LAKATOS, E.M.; MARCONI, M.A. Fundamentos de metodologia científica. $5^{a}$ ed. São Paulo: Atlas, 2003.

MOODY, D.; WALSH, P. Measuring the value of information: an asset valuation approach. In: EUROPEAN CONFERENCE ON INFORMATION SYSTEMS, 7., 1999, Copenhagen. existente; oficializar a atividade de validação da categorização das informações pelo arquivista da organização; usar como referência um Código de Classificação de Documentos para a classificação de todas as informações da organização; e divulgar constantemente, para toda a organização, o mapa de informações construído.

Conclui-se que apenas a consulta disponível em sistemas informatizados não é suficiente para ter acesso à informação. A construção de um mapa de informações permite facilitar a localização e o acesso às informações armazenadas nos diversos sistemas corporativos. O seu uso subsidiará os gerentes da área de tecnologia da informação e gestores de negócio da organização estudada, no atendimento às suas necessidades de informações no que diz respeito a localizálas na empresa e identificar a quais processos de negócio estão associadas.

Proceedings... Frederiksberg, Denmark: Business School, 1999.

MORESI, E.A.D. Apostila de metodologia da pesquisa. Brasília: Universidade Católica de Brasília, 2003.

PRUSAK, L; McGEE, J. Gerenciamento estratégico da informação: aumente a competitividade e a eficiência de sua empresa utilizando a informação como uma ferramenta estratégica. 11 . ed. Rio de Janeiro: Campus, 1994.

ROSENFELD, L; MORVILLE, P. Information architecture for the world wide web. $2^{\text {nd }}$ ed. [s.l.]: O'Reilly \& Associates, 2002.

ROSINI, A.M.; PALMISANO, A. Administração de sistemas de informação e a gestão do conhecimento. São Paulo: Pioneira Thomson Learning, 2003.

WETHERBE, J.C.; BRANCHEAU, J.C. Information architectures: methods and practice. Information Processing \& Management, v.22, n.6, p.453-463, 1986.

WETHERBE, J.C.; VOGEL, D.R. Information architecture: sharing the sharable resource. CAUSE From Cause/Effect, v.14, n.2, 1991. Available from: <http://www.educause.edu/ir/library/text/ CEM9122.txt>. Cited: 29 Mar. 2004.

WURMAN, R.S. Ansiedade de informação: como transformar informação em compreensão. São Paulo: Cultura Editores Associados, 1991.

ZACHMAN, J.A. The framework for enterprise architecture: background, description and utility. 1992. Available from: <http:/ /members. ozemail.com.au/ visible/paper/zachman3.htm>. Cited: 23 Aug. 2004. 
ANEXO 1

DESCRIÇÃO DOS SISTEMAS CORPORATIVOS DA EMBRAPA INCLUÍDOS NO ESPAÇO INFORMACIONAL MAPEADO

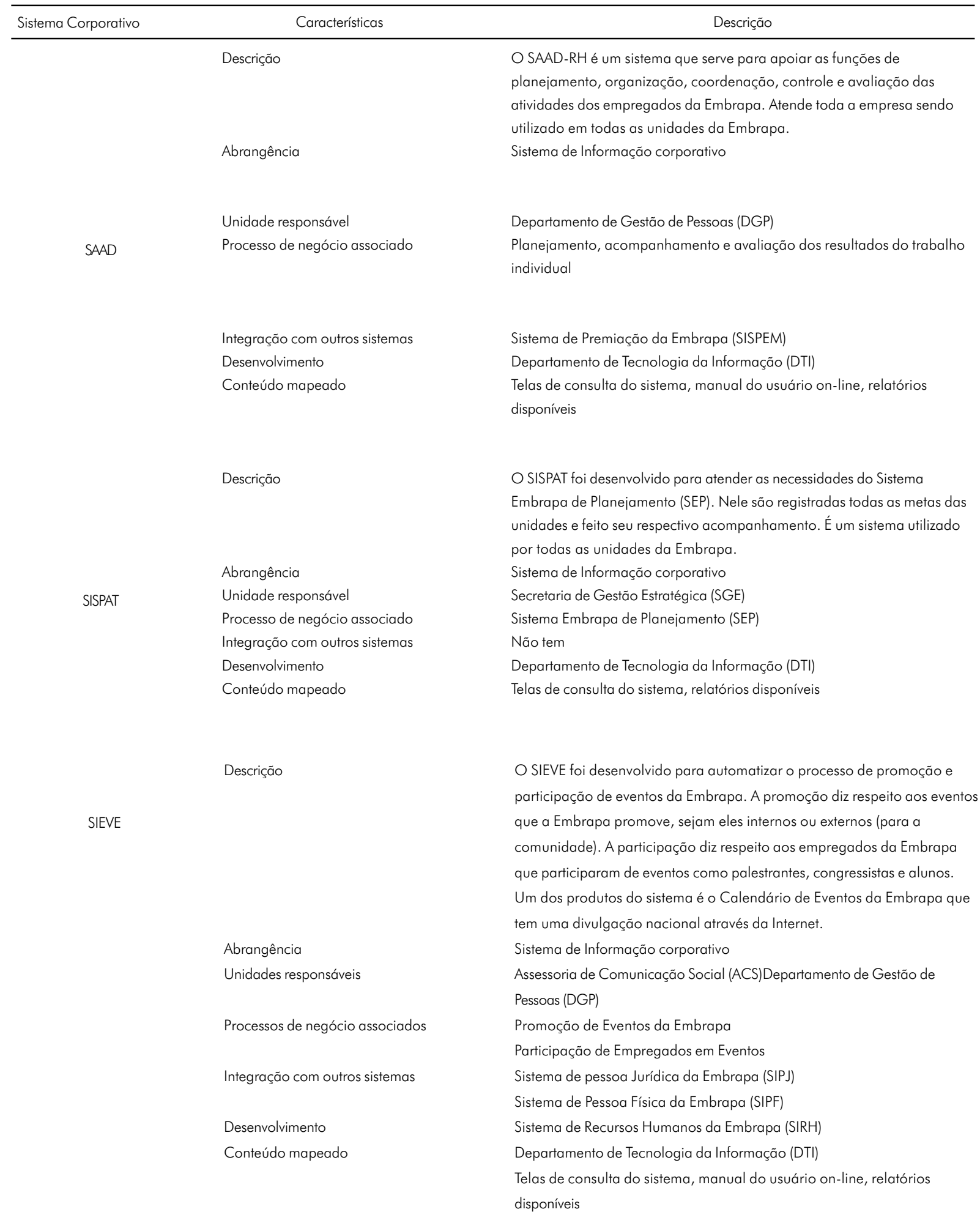

SAAD: sistema de planejamento, acompanhamento e avaliação de resultados do trabalho individual; SIEVE: sistema de eventos da Embrapa; SISPAT: sistema de planejamento de atividades da Embrapa; $\mathrm{RH}$ : recursos humanos. 
ANEXO 2

EXEMPLO DA ESTRUTURA FÍSICA DO MAPA DE INFORMAÇÕES COM UM CONTEÚDO MAPEADO

\begin{tabular}{|c|c|c|c|c|c|c|c|}
\hline AG & Pessoal & $\begin{array}{c}\text { Lotação de } \\
\text { pessoal }\end{array}$ & $\begin{array}{l}\text { Quais os empregados estão } \\
\text { subordinados aos superviso- } \\
\text { res de uma unidade, em um } \\
\text { determinado ano? }\end{array}$ & $\begin{array}{l}\text { Planejamento, Acompa- } \\
\text { nhamento e Avaliação } \\
\text { de Resultados do Traba- } \\
\text { lho Individual }\end{array}$ & DGP & $\begin{array}{l}\text { Sistema } \\
\text { SAAD }\end{array}$ & $\begin{array}{l}\text { Informação restrita ao } \\
\text { perfil de Chefe de Uni- } \\
\text { dade e representante do } \\
\text { SRH na Unidade }\end{array}$ \\
\hline$P \& D$ & $\begin{array}{c}\text { Sistema } \\
\text { Embrapa de } \\
\text { Planejamento }\end{array}$ & Institucional & $\begin{array}{l}\text { Quantas metas técnicas fo- } \\
\text { ram cumpridas pelas Uni- } \\
\text { dades descentralizadas de } \\
\text { acordo com o índice de } \\
\text { cumprimento de metas? }\end{array}$ & $\begin{array}{l}\text { Sistema Embrapa de } \\
\text { Planejamento }\end{array}$ & SGE & $\begin{array}{l}\text { Sistema } \\
\text { SISPAT }\end{array}$ & $\begin{array}{l}\text { Informação sem restrição } \\
\text { de acesso }\end{array}$ \\
\hline
\end{tabular}

AG: administração geral; P\&D: pesquisa e desenvolvimento; SGE: secretaria de gestão estratégica; DGP: departamento de gestão de pessoa; SAAD: sistema de planejamento, acompanhamento e avaliação de resultados do trabalho individual; SRH: sistema de recursos humanos; SISPAT: sistema de planejamento de atividades da Embrapa.

ANEXO 3

DEMONSTRATIVO CONSOLIDADO DAS INFORMAÇÕES MAPEADAS NA EMBRAPA, POR CLASSIFICAÇÃO DE INFORMAÇÃO

\begin{tabular}{|c|c|c|c|c|c|}
\hline \multirow[b]{2}{*}{ Classe } & \multirow[b]{2}{*}{ Subclasse } & \multirow[b]{2}{*}{ Subgrupo } & \multicolumn{3}{|c|}{ Quantidade } \\
\hline & & & Informação* & Relatório** & Consulta*** \\
\hline & & Lotação de pessoal & 2 & 2 & 1 \\
\hline & Pessoal & Classificação de função & 1 & 1 & 1 \\
\hline & & Reestruturação salarial & 27 & 26 & 3 \\
\hline & Organização e & & & & \\
\hline \multirow[t]{7}{*}{ Administração Geral } & Funcionamento & Regimentos e Estruturas & 1 & 0 & 1 \\
\hline & Finanças & Receita & 1 & 2 & 0 \\
\hline & Aperfeiçoamento e & Promovidos pela Instituição & 12 & 3 & 0 \\
\hline & Treinamento & Promovidos por outra instituição & 12 & 3 & 0 \\
\hline & Planos e programas e & Avaliação de Desempenho de & & & \\
\hline & Projetos de Trabalho & Unidades & 1 & 1 & 0 \\
\hline & & Projetos & 3 & 2 & 1 \\
\hline \multirow[t]{4}{*}{ Pesquisa e Desenvolvimento } & Sistema Embrapa de & & & & \\
\hline & Planejamento (SEP) & & & & \\
\hline & & Institucional & 12 & 9 & 0 \\
\hline & & Parcerias & 1 & 1 & 0 \\
\hline \multirow[t]{5}{*}{ Comunicação e Negócios } & Transferência de & Mecanismos para Transferência & & & \\
\hline & Tecnologia & de Tecnologia & 3 & 1 & 0 \\
\hline & & Capacitação & 1 & 2 & 0 \\
\hline & & Dia-de-campo & 1 & 2 & 0 \\
\hline & Solenidades, & & & & \\
\hline \multirow[t]{5}{*}{ Assuntos Gerais } & Comemorações e & & & & \\
\hline & Homenagens & Não tem subgrupo & 7 & 4 & 0 \\
\hline & Congressos, & & & & \\
\hline & Conferências, & & & & \\
\hline & Simpósios, Encontros & Não tem subgrupo & 7 & 4 & 0 \\
\hline Total & & & 92 & 63 & 7 \\
\hline
\end{tabular}

TransInformação, Campinas, 22(2):101-110, maio/ago., 2010 